\title{
Criptococosis diseminada con compromiso esplénico y meníngeo en una paciente con sida
}

\section{Disseminated cryptococcosis with splenic and meningeal involvement in a patient with AIDS}

\author{
Jairo lizarazo, Édgar Parra, Óscar Parada, Noé Castro, \\ Óscar Chaves, Yeni Peña • Cúcuta
}

\begin{abstract}
Resumen
Se presenta el caso de una mujer de 37 años de edad, con diagnóstico reciente de infección por VIH, que consultó por cefalea, diarrea, vómito y fiebre de dos semanas de evolución. Se encontraba caquéctica con una gran esplenomegalia. El estudio del líquido cefalorraquídeo demostró una meningitis por Cryptococcus neoformans. Las imágenes diagnósticas evidenciaron una gran esplenomegalia con lesiones focales. La biopsia percutánea con aguja cortante $\mathrm{N}^{\circ} 18$ de una de las lesiones focales confirmó la criptococosis esplénica. La paciente recibió tratamiento secuencial con anfotericina B y fluconazol con una evolución satisfactoria. Las lesiones esplénicas se resolvieron completamente y la paciente se encuentra actualmente asintomática y recibe profilaxis secundaria con fluconazol y terapia antirretroviral (Acta Med Colomb 2010; 35: 31-34).

Palabras clave: criptococosis, cryptococcus, infecciones oportunistas asociadas al síndrome de inmunodeficiencia adquirida, bazo, esplenomegalia, biopsia con aguja .
\end{abstract}

\begin{abstract}
We present the case of a 37-year-old woman with a recent diagnosis of HIV-infection. The patient consulted because of headache, diarrhea, vomit and fever of two weeks' evolution. The patient had a wasting syndrome, and severe splenomegaly. Cerebral spinal fluid study showed meningitis by Cryptococcus neoformans. CT-scan revealed splenomegaly with focal lesions. Percutaneous biopsy with 18 -gauge needle from one of the focal lesions confirmed cryptococcosis. The treatment was initiated with amphotericin B, followed by fluconazole. The patient's evolution has been satisfactory. Splenic lesions resolved and the patient is now asymptomatic and on secondary profilaxis with fluconazole and antiretroviral treatment (Acta Med Colomb 2010; 35: 31-34).

Keywords: cryptococcosis, cryptococcus, AIDS-related opportunistic infections, spleen, splenomegaly, needle biopsy.
\end{abstract}

Dr. Jairo Lizarazo: Neurólogo, Departamento de Medicina Interna, Hospital Universitario Erasmo Meoz, Cúcuta; Dr. Edgar Parra: Patólogo, Grupo de Patología, Instituto Nacional de Salud, Bogotá D.C.; Dres. Óscar Parada: Radiólogo, Departamento de Imágenes Diagnósticas; Dr. Noé Castro: Internista, Departamento de Medicina Interna; Dr. Óscar Chaves: Infectólogo, Unidad de Epidemiología; Yeni Peña: Licenciada en Enfermería, Coordinadora de la Unidad de Epidemiología. Hospital Universitario Erasmo Meoz, Cúcuta.

Origen de los recursos: para la elaboración de este trabajo se utilizaron recursos del Hospital Universitario Erasmo Meoz de Cúcuta y del Instituto Nacional de Salud de Bogotá D.C., Colombia.

Declaración de conflicto de interés: los autores declaramos no tener conflictos de interés.

Correspondencia: Dr. Jairo Lizarazo, Calle $13 \mathrm{~N}^{\circ} 1 \mathrm{E}-44$ consultorio 404B, Cúcuta, Colombia. Teléfono: 5722635 ,

E-mail: jflizar@gmail.com

Recibido: 13/XI/09 Aceptado: 4/I/2010

\section{Introducción}

La criptococosis es una micosis sistémica que afecta principalmente el sistema nervioso central (SNC) (1). Esta micosis continúa siendo, en los países en donde la cobertura de la terapia antirretroviral de gran actividad (TARGA) es baja, una de las principales infecciones oportunistas del SNC en los pacientes con sida, y en Colombia ocupa el segundo lugar (2). En países industrializados, en donde la TARGA es ampliamente disponible la incidencia de la criptococosis ha disminuido sensiblemente (3).

La esplenitis por Cryptococcus neoformans es rara y, usualmente, se presenta como parte de una criptococosis diseminada en pacientes inmusuprimidos, especialmente en aquellos que sufren del sida y, frecuentemente, es un hallazgo de autopsia (4).

A continuación describimos el caso de una mujer con sida que presentó criptococosis diseminada con compromiso esplénico y meníngeo, la cual tuvo una buena respuesta terapéutica.

\section{Presentación del caso}

Se trata de una mujer de 37 años de edad, natural de Salazar de las Palmas (Norte de Santander) y procedente de Bucaramanga, residenciada en Cúcuta en los dos últimos 
meses previos al ingreso hospitalario. Acudió al servicio de consulta externa de Infectología del Hospital Universitario Erasmo Meoz el 20/06/08 por presentar cefalea global, opresiva y persistente, vómito, diarrea y fiebre de dos semanas de evolución. Hacía un mes se le había confirmado el diagnóstico de infección por VIH (dos pruebas de Elisa y un Western Blot, todas positivas) y el 07/06/08 había iniciado terapia antirretroviral con efavirenz, abacavir y lamivudina.

Entre los antecedentes patológicos refirió una biopsia gástrica endoscópica (30/01/08) que demostró una gastritis antral crónica y, la presencia de colelitiasis y barro biliar en una ecografía abdominal total (01/03/08). Su historial obstétrico fue de G4P4A4C0, le habían realizado esterilización por la técnica de Pomeroy y apendicectomía. Entre los exámenes de laboratorio realizados antes del ingreso tenía como hallazgo positivo una IgG anti toxoplasma elevada $(46.4 \mathrm{UI} / \mathrm{mL}$ para un normal de 0.0 a $2.0 \mathrm{UI} / \mathrm{mL}), \mathrm{El}$ antígeno de superficie para la hepatitis B fue negativo. En los últimos meses había perdido peso (aproximadamente $13 \mathrm{Kg}$ en dos meses).

En el examen físico se encontró una mujer caquéctica con un peso de $32 \mathrm{~kg}$ (índice de masa corporal 14,2) con marcada palidez mucocutánea, con tensión arterial de 110/70 $\mathrm{mmHg}$, taquicárdica (frecuencia cardiaca de 120 por minuto) y taquipneica (frecuencia respiratoria de 28 por minuto) y afebril (temperatura axilar de $37^{\circ} \mathrm{C}$ ). La auscultación cardiopulmonar no evidenció alteraciones. En el abdomen se palpó el bazo aumentado de tamaño $13 \mathrm{~cm}$ por debajo de la reja costal izquierda, de superficie lisa y doloroso. En el examen ginecológico se evidenció una leucorrea sugestiva de una candidiasis vaginal.

La paciente fue hospitalizada ese mismo día y se solicitaron exámenes de laboratorio cuyos resultados están consignados en la Tabla 1. El hallazgo anormal más importante fue la pancitopenia. También se solicitó una ecografía abdominal total la cual informó como único hallazgo patológico una esplenomegalia con lesiones focales hipoecogénicas. Luego, se realizó una tac abdominal (Figura 1) que demostró una gran esplenomegalia con lesiones hipodensas focales, que no captaban el medio de contraste.

El 23/06/08 presentó un cuadro confusional con disartria que se resolvió en pocas horas. El 25/06/08 se le practicó una punción lumbar diagnóstica. Los hallazgos del LCR (Tabla 1) fueron característicos de una criptococosis meníngea, la cual se confirmó posteriormente con un cultivo, en el que se recuperó Cryptococcus neoformans, sensible a fluconazol y voriconazol; no se reportó sensibilidad a la anfotericina (Corporación para Investigaciones Biológicas, Medellín). Con este diagnóstico se le inició anfotericina B intravenosa a dosis de $0.7 \mathrm{mg} / \mathrm{kg} / \mathrm{día}$.

La paciente requirió transfusión de cuatro unidades de glóbulos rojos. Luego de mejorar sus condiciones generales y, especialmente, la trombocitopenia, el 14/07/08 se le realizó una biopsia esplénica por punción con aguja $\mathrm{N}^{\circ}$ 18 de una de las lesiones focales, guiada por ecografía, la

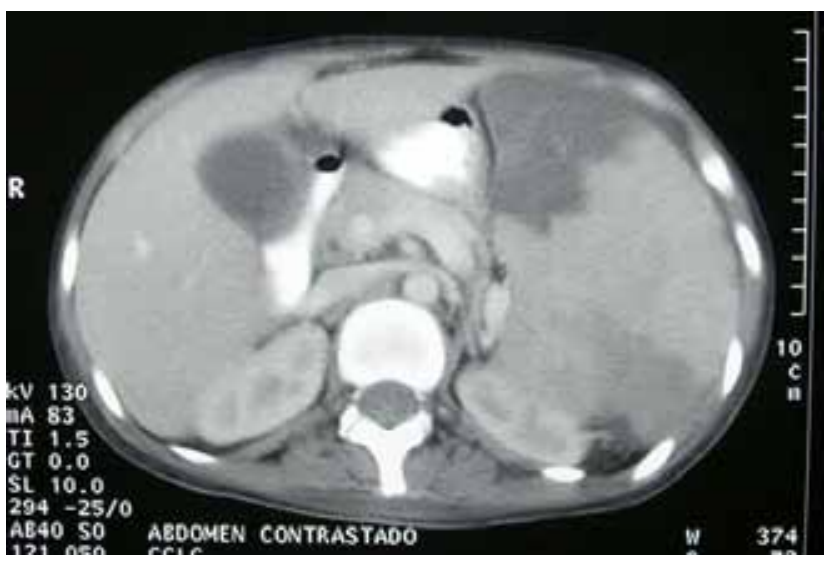

Figura 1. TAC de abdomen. Esplenomegalia masiva con presencia de lesiones focales hipodensas, más evidentes hacia el polo anterior.

cual no tuvo complicaciones. El estudio histopatológico, que incluyó coloraciones especiales para hongos (Figuras 2-5) confirmó la criptococosis esplénica. El cultivo de la biopsia fue negativo para hongos. Una prueba de látex para Cryptococcus en suero fue reactiva a una dilución $\geq$ 1:1024.

Tabla 1. Exámenes paraclínicos.

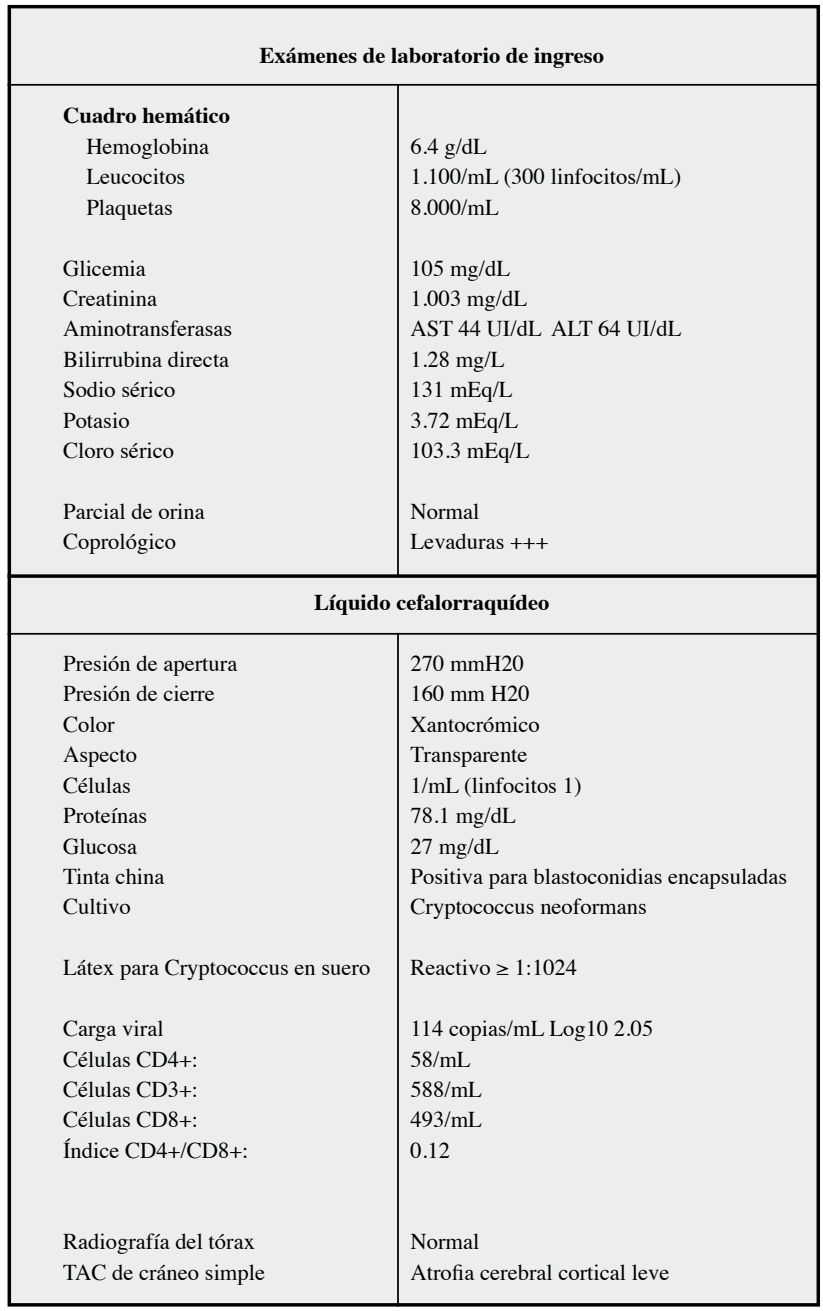



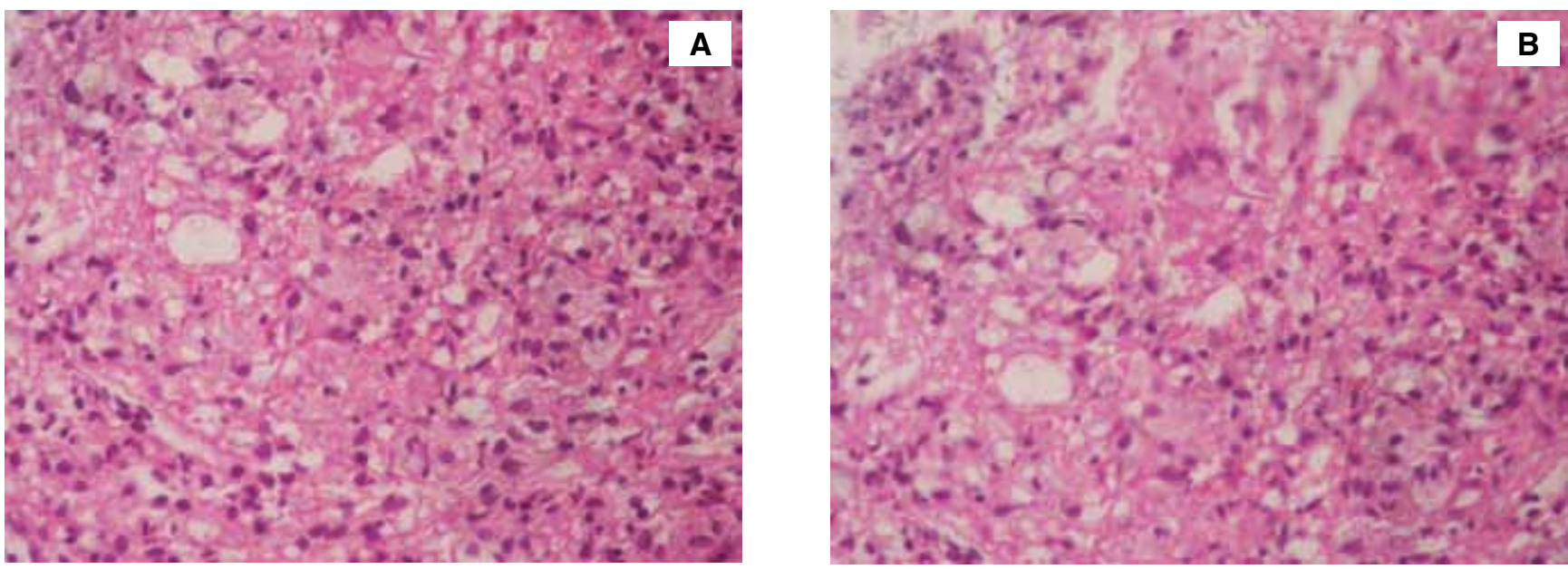

Figura 2 (a y b). Biopsia esplénica (H\&E 60x y 100x): nótese la escasa respuesta inflamatoria y la presencia de las blastoconidias.
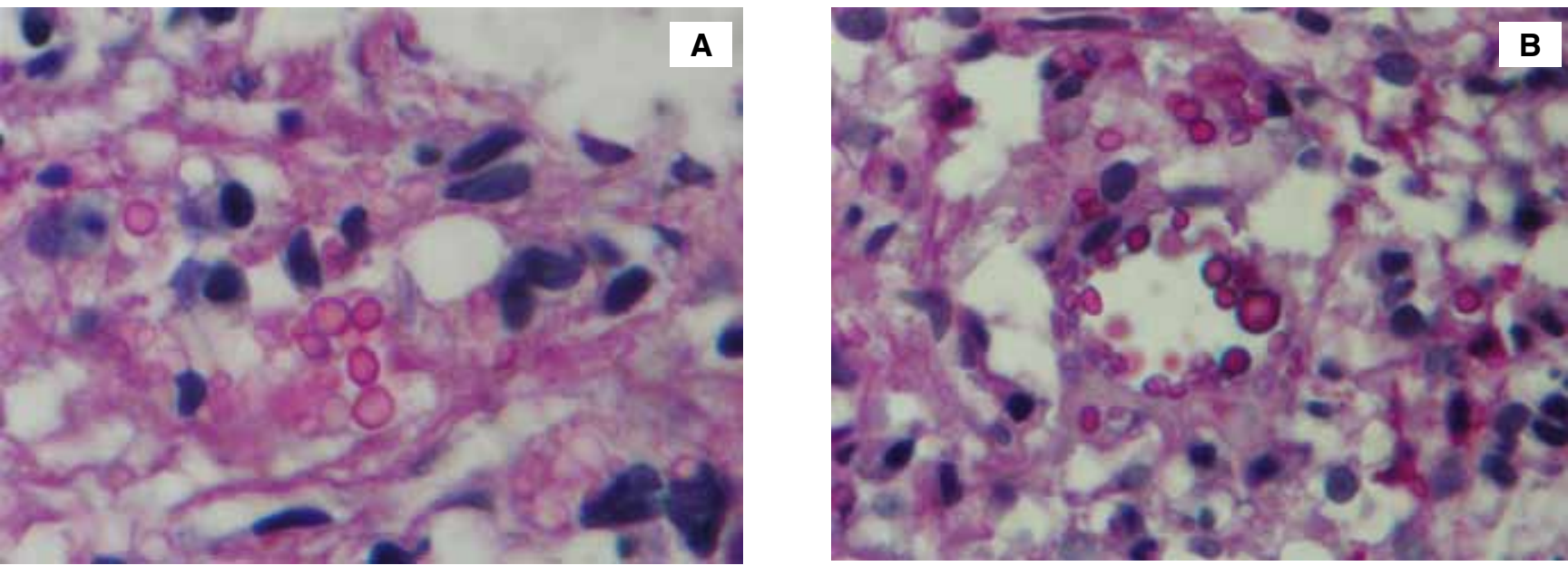

Figura 3 (a y b). Biopsia esplénica (PAS 100x): blastoconidias algunas de ellas gemantes de C. neoformans.

El número de células CD4+ fue de 58/mL y la carga viral de 114 copias $/ \mathrm{mL}$. La paciente recibió una dosis total de anfotericina B de $635 \mathrm{mg}$ en un lapso de 22 días, luego se continuó con fluconazol $400 \mathrm{mg}$ diarios por ocho semanas y actualmente recibe fluconazol $200 \mathrm{mg}$ diarios.

La paciente fue mejorando paulatinamente y el 16/07/08 se envió a su hogar. El 20/07/08 consultó por urgencias debido a dolor en el hipocondrio izquierdo. Se encontró la esplenomegalia de menor tamaño, la cual no evidenciaba lesiones focales en la ecografía de control.

La evolución en los meses siguientes fue hacia la mejoría. La última consulta fue el 27/08/09 y se encontró una paciente en buenas condiciones generales, trabaja en oficios domésticos, aumentó de peso $(50 \mathrm{Kg})$, sin esplenomegalia y con un examen neurológico normal. En la última evaluación (26/08/09) tenía una carga viral indetectable, células CD4+: 176/mL, CD3+: 1607/mL, CD8+: 132/mL7, índice
CD4+/CD8+: 0,13. Recibe terapia antirretroviral (efavirenz, zidovudina y lamivudina) y la profilaxis secundaria para criptococosis con fluconazol, además de trimetropínsulfametoxazol.

\section{Discusión}

La criptococosis del bazo es rara. En la Encuesta Nacional realizada por el Grupo Colombiano para el Estudio de la Criptococosis durante los años 1997-2005 de un total de 931 casos no se informó en ninguno de ellos afectación esplénica (5). Usualmente, esta presentación clínica se da en el curso de una criptococosis diseminada y con mayor frecuencia en los pacientes inmunocomprometidos $(4,6)$, aunque ocasionalmente se ha descrito en pacientes $\mathrm{VIH}$ negativos (7).

En esta paciente llama la atención la gran esplenomegalia que motivó la consulta. Sin embargo, fueron las manifestacio- 
nes clínicas neurológicas la que permitieron el rápido diagnóstico de la criptococosis mediante el estudio del LCR.

El abordaje diagnóstico de las lesiones esplénicas se demoró en esta paciente debido a la grave trombocitopenia. Una vez mejorada ésta, la biopsia por punción con aguja guiada por ecografía permitió el diagnóstico histopatológíco de la micosis. Es entendible que el cultivo de la biopsia fuera negativo debido al tiempo que ya llevaba recibiendo anfotericina B.

Históricamente, los procedimientos percutáneos del bazo guiados por imagen han sido evitados debido al temor de un riesgo elevado de complicaciones. Entre ellas están la hemorragia primaria y la punción inadvertida del colon, la pleura y el riñón (8). La negativa a realizar aspiraciones esplénicas con aguja proviene de percepciones infundadas acerca del peligro que existe de un gran riesgo de hemorragia. La creencia de que el muestreo esplénico es inseguro no está soportada por ninguna estadística. Aunque, como en todo procedimiento invasor, las complicaciones pueden ocurrir, las tasas de complicaciones de la biopsia esplénica son similares a las de otros órganos abdominales. Una biopsia del bazo bajo guía de ultrasonido puede realizarse en forma segura, aún en niños (9). Un estudio de 47 biopsias esplénicas demostró que las agujas $\mathrm{N}^{\circ} 18$ tienen la tasa diagnóstica más alta comparada con las agujas $\mathrm{N}^{\circ} 21$, sin aumentar el número de complicaciones, y por eso es recomendable el uso de la aguja $\mathrm{N}^{\circ} 18$ cortante para la biopsia guiada por ultrasonido de lesiones esplénicas (10).

El diagnóstico diferencial de las lesiones focales del bazo incluye: quistes, abscesos, infartos, hematomas, lesiones tumorales primarias benignas y malignas, siendo el linfoma la más frecuente entre las malignas, y metástasis (11).

En casos de criptococosis esplénica extensa se ha informado ausencia de respuesta inflamatoria en el bazo en presencia de los microorganismos, como sucedió en nuestro caso (4). Como hallazgos patológicos adicionales se ha descrito disminución de la pulpa blanca, vasos sanguíneos escleróticos y aumento de los depósitos de colágeno y de los histiocitos, además de engrosamiento de la cápsula (4). La depleción de la pulpa blanca es una anormalidad atribuida directamente a la infección por el VIH y se cree que representa una grave reducción en el número de células $\mathrm{T}$ y una alteración en la función de los macrófagos (12).
No obstante la gran esplenomegalia, el tratamiento de la criptococosis con el esquema secuencial de inicio con anfotericina $\mathrm{B}$ y luego con fluconazol, permitió una rápida mejoría clínica de la paciente y la desaparición de las lesiones esplénicas en pocas semanas.

Como conclusión, ante la presencia de esplenomegalia con lesiones focales en las imágenes diagnósticas en un paciente inmunosuprimido, se debe tener en cuenta a la criptococosis como diagnóstico diferencial, en mayor medida si existen manifestaciones clínicas del SNC.

\section{Agradecimientos}

A Elizabeth Castañeda por su invaluable ayuda en la evaluación crítica del manuscrito. A Catalina de Bedout de la CIB por la realización de los estudios micológicos.

\section{Referencias}

1. Jarvis JN, Harrison T. HIV-associated cryptococcal meningitis. AIDS 2007; 21: 2119-29.

2. Lizarazo J. Infecciones oportunistas del sistema nervioso central en sida. Acta Neurol Colomb 2007; 23: 286-7.

3. Mirza SA, Phelan M,Rimland D, Graviss E,Hamill R, Brandt ME, et al. The changing epidemiology of cryptococcosis: an update from population-based active surveillance in 2 large metropolitan areas, 1992-2000. Clin Infect Dis 2003; 36: 789-94.

4. Viriyavejakul P, Rojanasunan P, Viriyavejakul A, Tangwanicharoen T, Punyarit P, Punpoowong B, et al. Necropsy in HIV-infected patients. Southeast Asian J Trop Med Public Health 2002; 33: 85-91.

5. Lizarazo J, Linares M, de Bedout C, Restrepo A, Agudelo CI, Castañeda $\mathbf{E}$ y Grupo Colombiano para el estudio de la criptococosis. Estudio clínico y epidemiológico de la criptococosis en Colombia; resultados de nueve años de la encuesta nacional, 1997-2005. Biomédica 2007; 27: 94-109.

6. Coskun ZU, Mathews D, Weatherall P, Skiest D, Öz OK. Cryptococcal lymphadenitis and massive splenomegaly in an immunocompromised patient. Clin $\mathrm{Nucl}$ Med 2007; 32: 314-6.

7. Muslimani A, Francis NS, Gopalakrishna KV, Daw HA. Disseminated cryptococcosis in a patient with idiopathic CD4+ T-lymphocytopenia. Clin Adv Hematol Oncol 2008; 6: 446-8.

8. Lucey BC, Boland GW, Maher MM, Hahn PF, Gervais DA, Mueller PR. Percutaneous nonvascular splenic intervention: a 10-year review. American Journal of Roentgenology 2002; 179: 1591-6.

9. Muraca S, Chait PG, Connolly BL, Baskin KM, Temple MJ. US-guided core biopsy of the spleen in children. Radiology 2001; 218: 200-6.

10. Liang P, Gao Y, Wang Y, Yu X, Yu D, Dong B. US-guided percutaneous needle biopsy of the spleen using 18-gauge versus 21-gauge needles. J Clin Ultrasound 2007; 35: 477-82.

11. Kamaya A, Weinstein S, Desser TS. Multiple lesiones of the spleen: differential diagnosis of cystic and solid lesiones. Semin Ultrasound CT MR. 2006; 27: 389403.

12. Díaz LK, Murphy RL, Phair JP, Variakojis D. The AIDS autopsy spleen; comparison of the pre-anti-retroviral and highly active anti-retroviral therapy eras. Mod Pathol 2002; 15: 406-12. 